\title{
Physical forces during collective cell migration
}

\author{
Xavier Trepat ${ }^{1,2 \star}$, Michael R. Wasserman ${ }^{1}$, Thomas E. Angelini ${ }^{3}$, Emil Millet ${ }^{1}$, David A. Weitz ${ }^{3}$, \\ James P. Butler ${ }^{1,4}$ and Jeffrey J. Fredberg ${ }^{1 \star}$
}

Fundamental biological processes including morphogenesis, tissue repair and tumour metastasis require collective cell motions ${ }^{1-3}$, and to drive these motions cells exert traction forces on their surroundings ${ }^{4}$. Current understanding emphasizes that these traction forces arise mainly in 'leader cells' at the front edge of the advancing cell sheet ${ }^{5-9}$. Our data are contrary to that assumption and show for the first time by direct measurement that traction forces driving collective cell migration arise predominately many cell rows behind the leading front edge and extend across enormous distances. Traction fluctuations are anomalous, moreover, exhibiting broad nonGaussian distributions characterized by exponential tails ${ }^{10-12}$. Taken together, these unexpected findings demonstrate that although the leader cell may have a pivotal role in local cell guidance, physical forces that it generates are but a small part of a global tug-of-war involving cells well back from the leading edge.

The single adherent cell moves by the action of two synchronized cycles, one involving extension and contraction of its cytoskeleton and the other involving formation and detachment of its adhesions ${ }^{13,14}$. Although this complex process remains a matter of intense research ${ }^{14-16}$, it is now well established that a fundamental aspect of the motility mechanism is the transmission of contractile forces to the surrounding matrix at the cell's leading and trailing edges $^{17,18}$. In contrast with the case of migration of the single cell studied in isolation ${ }^{14-16}$, the case of collective migration of cells within a contiguous cell sheet has more physiological relevance but is substantially less well understood ${ }^{19}$. Within an advancing epithelial cell sheet, for example, each individual cell is physically constrained by its neighbours, and cell-cell signalling through biochemical and biophysical pathways may influence the collective motion of the group ${ }^{20,21}$. Do leader cells at the advancing front edge of the sheet exert physical forces locally that are transmitted rearward, from cell-to-cell, and thus act to pull along those cells in the ranks behind $d^{5,6,8,9}$ ? Or instead is each individual cell in the sheet mechanically self-propelled ${ }^{21}$ ? Or does cell proliferation expand the cell colony and thereby push the advancing front forward? Or is the correct answer none of the above? For more than a century these fundamental questions have been debated intensively ${ }^{5,22}$ and, using a variety of methods in vivo ${ }^{23}$, in vitro ${ }^{4,9,24}$ and in silico ${ }^{21}$, much conflicting evidence has accumulated. This conflicting evidence has been in most cases indirect or inferential, however, because within the cell sheet the physical forces themselves have remained largely inaccessible to direct experimental observation.

Here, we report by direct measurement the first explicit maps of those physical forces and their distribution. To do this within an advancing cell sheet, we used Fourier-transform traction microscopy together with a balance of forces that is demanded by straightforward application of Newton's laws of motion (see Supplementary Information S2 and S3). To address the case of an advancing cell sheet, however, traction microscopy as described originally ${ }^{18,25}$ or as modified subsequently ${ }^{26-28}$ is inadequate and therefore required fundamental reformulation (see Supplementary Information S2 and S3). We seeded a small number of Madin-Darby canine kidney epithelial cells $(\sim 5,000)$ at the centre of a soft collagen-coated polyacrylamide gel (Young's modulus of $1.3 \mathrm{kPa}$ ). The cells adhered readily to this substrate and within $24 \mathrm{~h}$ formed a confluent colony. With time, the colony expanded radially outward, thus providing a simple model of collective migration without need of damaging the monolayer as in classical scratch-wounding experiments (see Supplementary Information S1). Growth of the colony was largely insensitive to the stiffness of the underlying substrate (see Supplementary Information S4). After allowing the colony to expand for at least $72 \mathrm{~h}$, we mapped the traction forces that marginal and submarginal cells exerted on their underlying matrix.

We first assessed the locus of traction forces in the proximity of the leading edge (Fig. 1, Supplementary Movie S1). Maps of tractions normal $\left(T_{\perp}\right)$ and parallel $\left(T_{\|}\right)$to the leading edge show that tractions are not restricted to cells at the leading edge or even to cells located 2-3 rows behind it, as is commonly emphasized ${ }^{5,6,29}$. Instead, large tractions are applied by cells many cell rows behind the edge. Independent of the distance from the edge, both $T_{\perp}$ and $T_{\|}$exhibited broad non-Gaussian distributions characterized by exponential tails (Fig. 2a, b). The distribution of $T_{\perp}$ was skewed towards positive tractions at the leading edge, whereas the distribution of $T_{\|}$was symmetric. Both traction distributions narrowed as the distance from the leading edge increased. Taken together, these data are inconsistent with the existence of two populations of cells, each with a distinct mechanical phenotype, one corresponding to active mechanical leaders at the leading edge and the other to passive mechanical followers. Instead, our data show a single distribution, the tails of which were roughly exponential rather than Gaussian, revealing probabilities of high tractions much larger than would be predicted according to the central limit theorem for independent and identically distributed random variables. Exponential distributions have previously been reported at the level of the single focal adhesion ${ }^{30-32}$, thereby indicating that this particular kind of distribution might underlie tissue behaviour over multiple length scales.

Submarginal cells have previously been shown to extend cryptic lamellipodia beneath cells in front of them ${ }^{24}$. Regardless of the extent to which cryptic lamellipodia are mechanically active and represent a locus of force generation, traction forces generated by these submarginal cells are seen to be comparable to those at the leading edge. A more important question, however, is whether these

\footnotetext{
${ }^{1}$ Program in Molecular and Integrative Physiological Sciences, School of Public Health, Harvard University, Boston, Massachusetts 02115, USA, ${ }^{2}$ Unitat de Biofísica i Bioenginyeria, Universitat de Barcelona, Institute for Bioengineering of Catalonia, and Ciber Enfermedades Respiratorias, 08036 Barcelona, Spain, ${ }^{3}$ School of Engineering and Applied Sciences, Harvard University, Cambridge, Massachusetts 02138, USA, ${ }^{4}$ Dept. Medicine, Harvard Medical School, Boston, Massachusetts 02115, USA. *e-mail: xtrepat@ub.edu; jeffrey_fredberg@hsph.harvard.edu.
} 

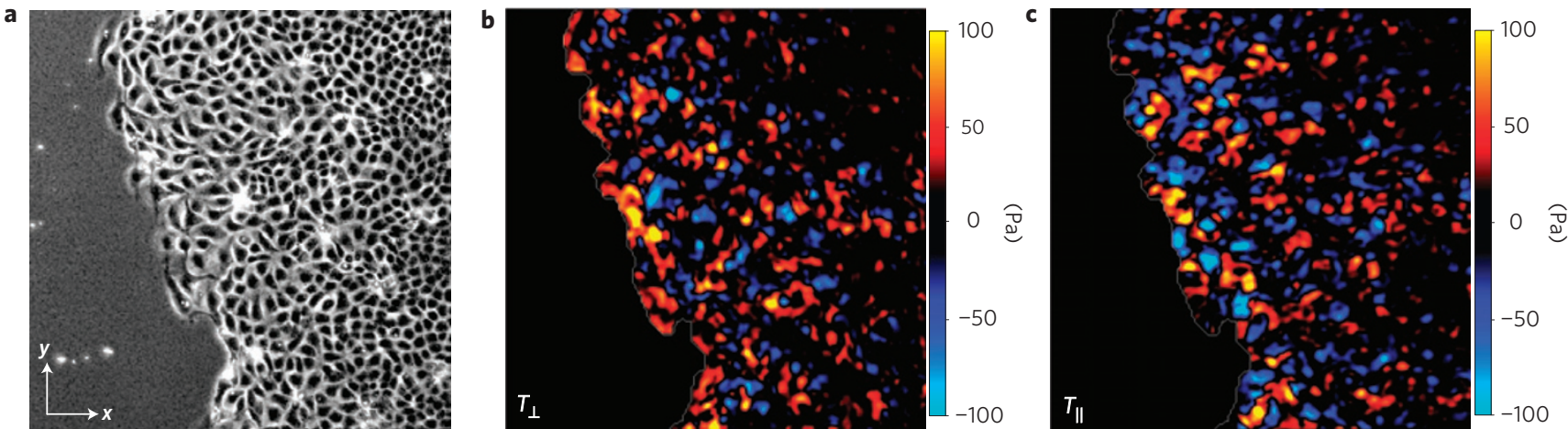

Figure 1 | Traction forces generated by a collectively migrating cell sheet. $\mathbf{a}$, Phase contrast image. $\mathbf{b}$, Tractions normal to the edge. $\mathbf{c}$, Tractions parallel to the edge. The field of view is $750 \mu \mathrm{m} \times 750 \mu \mathrm{m}$. $T_{\|}$and $T_{\perp}$ were calculated from $T_{x}$ and $T_{y}$ and from the local normal vector to the cell edge (see Supplementary Methods).
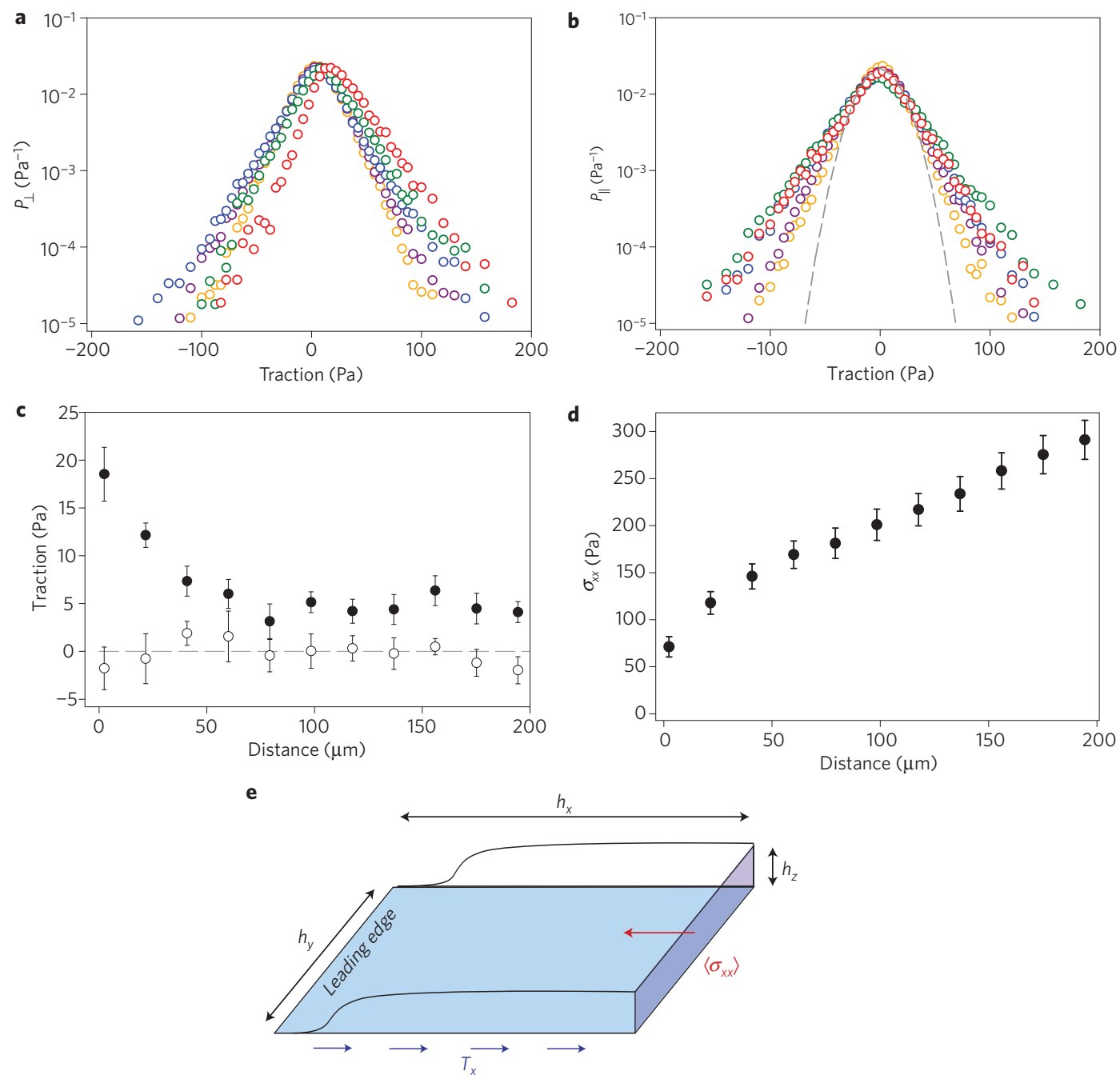

Figure 2 | Traction force distributions at different distances from the leading edge. a, Normal tractions. b, Parallel tractions. Red circles: first cell row from the leading edge; green circles: row 2; blue circles: rows 3-5; purple circles: rows 6-11; orange circles: rows 12-17. For computational simplicity, each row was assumed to be $19.2 \mu \mathrm{m}$ in radial dimension. Data were pooled from $n=4$ different cell sheets at four different time points for each well. The tails of each distribution appear straight in a semilog plot, showing the exponential nature of the distributions. A Gaussian fit to the distribution of parallel tractions for rows 12-17 is plotted as a reference (dashed grey line). c, The average normal traction decayed slowly with distance from the edge (filled symbols), whereas the average parallel traction was negligible and independent of the distance from the edge (open symbols). Error bars indicate standard errors. d, Stress within the cell sheet increased as a function of the distance from the leading edge. Error bars indicate standard errors. e, Schematic diagram illustrating the computation of stress within the cell sheet. The average stress $\left\langle\sigma_{x x}\right\rangle$ normal to a plane perpendicular to the substrate and parallel to the leading edge can be calculated by integration of tractions $T_{x}$ between the edge and the plane. 


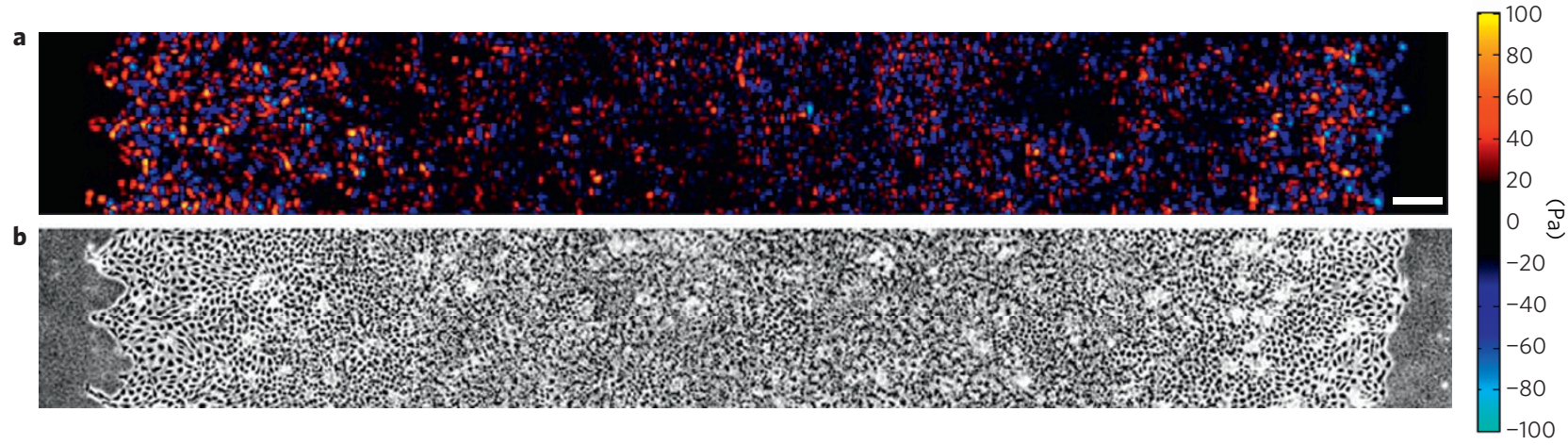

c

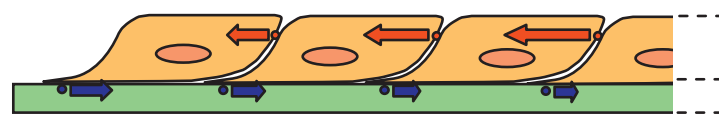

d

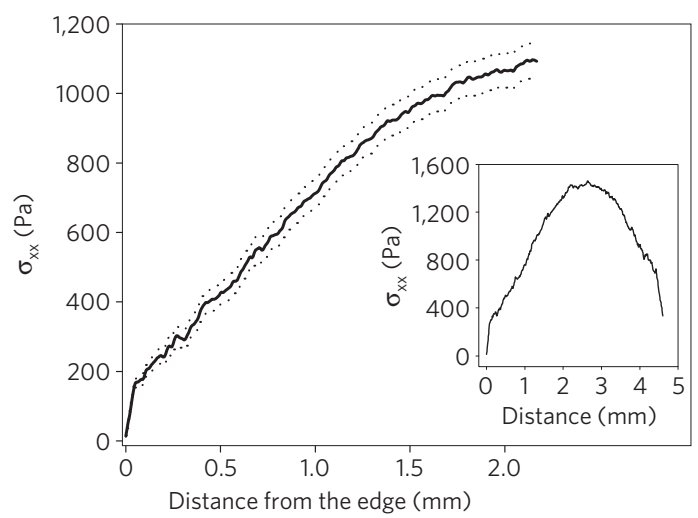

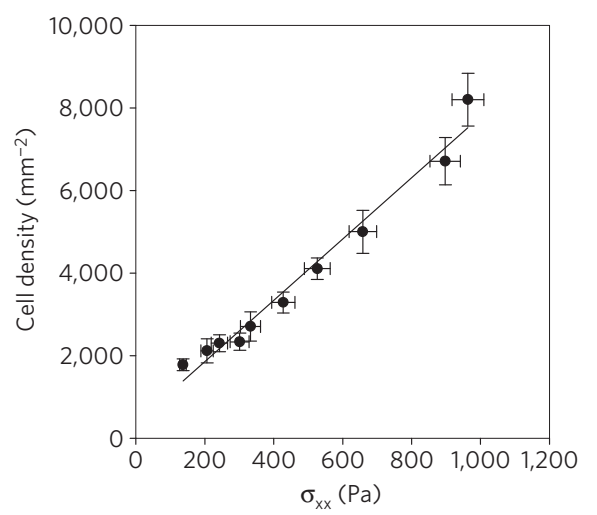

Figure $\mathbf{3}$ | The state of stress of the whole expanding colony is set by a global tug-of-war. a, Radial component of tractions along a diameter of the expanding cell colony $(\mathrm{bar}=200 \mu \mathrm{m})$. $\mathbf{b}$, Phase contrast image. Note the presence of multicellular protrusions on the left margin of the colony. This particular shape is reminiscent of that caused by fingering instabilities in fluids ${ }^{9}$. $\mathbf{c}$, Tug-of-war model. A small portion of the traction that each cell generates is transmitted to the cell behind. As such, tension in the cytoskeleton and cell-cell junctions increases towards the centre of the cell colony (red arrows). d, Once integrated over many cell rows, this small portion becomes dominant over traction fluctuations (see Fig. 2). Dotted lines indicate mean \pm standard error. Inset: Representative measurement of $\sigma_{x x}$ over the whole diameter of a colony. $\sigma_{x x}$ reaches a maximum roughly at the centre of the colony. The fact that $\sigma_{x x}$ does not decrease exactly to zero at the end of the monolayer points to the existence of weak shear stresses that also contribute to the force balance. e, The ratio between radial stress and cell density across the monolayer was roughly constant. Error bars indicate standard errors.

forces are balanced locally, as if each cell were self-propelled, or whether they are transmitted from cell to cell in a cooperative manner. We note that no amount of kinematic data or structural data, no matter how detailed, and no molecular manipulation, no matter how sophisticated, can ever suffice to resolve this question. Instead, we provide here a conclusive answer based solely on the application of Newton's laws. We begin by computing the spatial averages (denoted by \langle\rangle ) of $T_{\perp}$ and $T_{\|}$(Fig. 2c). As expected by symmetry, $\left\langle T_{\|}\right\rangle$was near zero. $\left\langle T_{\perp}\right\rangle$ was maximum at the leading edge and progressively decayed with distance from the edge before changing sign in the opposite half of the sheet. In contrast to $\left\langle T_{\|}\right\rangle$, however, the spatial average $\left\langle T_{\perp}\right\rangle$ far away from the edge remained weakly but systematically greater than zero, with typical tugging tractions of the order of $5 \mathrm{~Pa}$. In the context of cell mechanics, a regional stress of magnitude in this range is often regarded as being small, and is certainly small compared with the fluctuations that we observed. One might therefore conclude that its effects are essentially negligible; we come to a quite different conclusion, however.

Within the field of measurement-here spanning less than half the sheet diameter-the average stress at the interface between the cell base and the cell substrate, $\left\langle T_{\perp}\right\rangle$, was regionally positive everywhere. This stress acts systematically in a direction that pulls the sheet towards the leading edge. The question then arises, how are these stresses balanced, as is required by Newton's laws? The simple and inescapable answer is this: at any arbitrary given distance $L$ remote from the leading edge, the sum of the traction stresses perpendicular to the edge from $x=0$ up to $x=L$ must be balanced by forces carried within the cell sheet at position $L$ (Fig. 2e). At every position within the sheet, therefore, the accumulated traction must be balanced by local cell-borne stresses that are transmitted along the cell sheet by the cytoskeleton within cells and by cell-cell junctions between cells. Using $\sigma_{x x}$ to denote stresses within the cell sheet on a plane perpendicular to the substrate and parallel to the edge, as distinct from cell tractions $T$ at the cell-substrate interface, force balance demands that these be related by

$$
\left\langle\sigma_{x x}(x)\right\rangle=\frac{1}{h_{z} h_{y}} \int_{0}^{x} \int_{0}^{h_{y}} T_{x}\left(x^{\prime}, y^{\prime}\right) \mathrm{d} x^{\prime} \mathrm{d} y^{\prime}
$$

where we take cell height, $h_{z}$, to be roughly $5 \mu \mathrm{m}$ (ref. 33), and $h_{y}$ is the length of the field of view.

We note, first, that if each cell were entirely self-propelled, then $\left\langle\sigma_{x x}\right\rangle$ would be identically zero everywhere. This possibility can now be ruled out (Fig. 2 d). Instead, $\left\langle\sigma_{x x}\right\rangle$ increased steadily with 

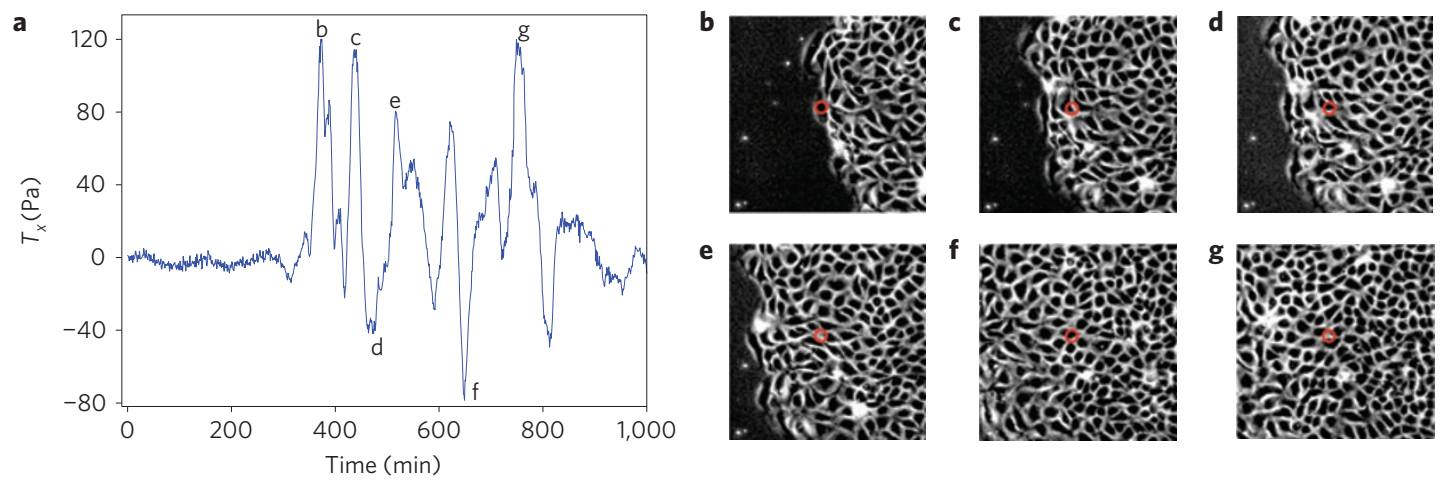

Figure 4 | Time fluctuations of tractions show cell-cell force transmission. $\mathbf{a}$, Traction $T_{x}$ at a fixed point of the gel (red circle in $\mathbf{b}-\mathbf{g}$ ) as cells crawl over that point. $\mathbf{b}-\mathbf{g}$, The right-to-left advance of the cell sheet at the time points labelled in $\mathbf{a}$. Note that $T_{x}$ remains mostly positive throughout the observation period, which implies stress transmission. (See Supplementary Movie S2.)

$x$, reaching a stress $\left\langle\sigma_{x x}\right\rangle \sim 300 \mathrm{~Pa}$ within the first 10 cell rows (Fig. 2d). Second, although they contribute little to the overall balance of forces, the pull that leading cell rows exert may be sufficient to direct the ranks immediately behind; in that sense they may still be 'leaders'. To study further the spatial extent of this tugging mechanism of force transmission, we obtained traction maps spanning the whole diameter of the cell colony (Fig. 3). Approaching the centre of the cell colony, $\left\langle\sigma_{x x}\right\rangle$ increased steadily and reached values that dominated traction fluctuations. Therefore, as the cell sheet grows, it exists in a global state of tensile stress. Such tensile stress establishes definitively that a build-up of pressure caused by proliferation is not the driving mechanism that underlies expansion of the cell sheet, for if this were the case, stresses within the monolayer would be compressive and tractions would point outwards. In both regards, we found systematic evidence to the contrary. Furthermore, our data show that cell density increased proportionally to the tensile stress $\sigma_{x x}$; for reasons that remain unclear, proliferation during tissue growth seems to be regulated in such a way that the ratio between density and tensile stress is invariant (Fig. 3e). Shraiman ${ }^{34}$ proposed that lateral stresses may act as a local feedback signal to regulate the rate of tissue growth, although his model deals with monolayer compression and buckling as opposed to the tensile stresses applicable here.

To assess further the nature of force transmission, we considered the time evolution of tractions at specific points of the traction map. An illustrative example of such time evolution is shown in Fig. 4 (see also Supplementary Movie S2). As the first leader cell of the sheet advanced over the traction sensing point (that is, a particular pixel of the traction map), a strong pull was first observed followed by a sharp decay. This decay, however, did not drop significantly below zero as would be the case for a self-propelled cell in isolation. Instead, a second sharp pulse was observed with a maximum corresponding to the boundary between the third and fourth cells. In this particular case, the first significantly negative force was observed after the fourth row, implying that the force generated by the first three rows was almost entirely transmitted to the following rows. In every case, traction fluctuations occurred at timescales longer than the time for a whole cell to move one cell length, which further demonstrates the existence of mechanical cooperativity and force transmission at length scales larger than the single cell.

In this connection, an exponential distribution of forces as reported here has been considered to be the signature of the force distributions that arise in jammed granular materials such as a pile of sand, grain in a silo or coffee beans stuck in a chute ${ }^{10-12}$, although the precise asymptotic form remains an open question ${ }^{35}$. The physics of jammed materials remains poorly understood, but the exponential nature of the force distribution is thought to arise from the combination of three generic features: close packing, structural disorder and long-range force transmission ${ }^{36}$. Both close packing and structural disorder are obvious properties of the cell sheet (Supplementary Movies S1 and S2), and here we provide evidence that transmission of force is long-ranged with exponentially distributed tails. Mechanics of jammed granular matter is governed by compressive stresses, however, whereas that of the cell sheet is governed by tensile stresses as demonstrated here. Although the connection between them remains a matter of speculation, the analogy between jammed inert materials and collective migration of cells is striking and suggests that these systems may share common mechanisms of long-range force transmission that are yet to be fully understood.

In summary, we present here definitive evidence establishing that collective motion in an advancing epithelial cell sheet results neither from leader cells dragging those behind, nor from cells that are individually self-propelled. Instead, each individual cell, both at the leading edge and well inside the sheet, engages in a global tug-of-war that integrates local force generation into a global state of tensile stress. Such a mechanism is innately integrative and would be inapparent in any cell studied in isolation. Whether this integrative mechanism is specific to certain tissues or instead is applicable generally during development, tissue healing and disease remains an open question, but one that is now accessible to direct experimental attack.

\section{Methods}

Cell culture. Madin-Darby canine kidney cells (strain II) were cultured on plastic flasks in MEM with Earle's salts supplemented with 5\% FBS, 2 mM L-glutamine, $100 \mathrm{U} \mathrm{ml}^{-1}$ penicillin and $100 \mu \mathrm{g} \mathrm{ml}^{-1}$ streptomycin. To seed a cell colony for experiments, a $5 \mu \mathrm{l}$ drop of supplemented media containing 5,000 cells was added to the centre of the gels. Surface tension limited the drop to approximately $1 \mathrm{~mm}^{2}$ of the central region of the gel. The cells were allowed to adhere to the gel for $30 \mathrm{~min}$ at $37^{\circ} \mathrm{C}$ and $5 \% \mathrm{CO}_{2}$ before $2 \mathrm{ml}$ of supplemented media was added to cover the whole surface of the dish.

Experiments. All experiments were conducted 3-4 days after seeding the cells. Three different images were collected every $60 \mathrm{~s}$, one imaging cells in phase contrast, one imaging the layer of beads located immediately below the cells and one imaging the diffraction rings of the layer of beads attached to the glass underlying the gel (Supplementary Information S2). A typical experiment lasted 6-24 h. At the end of the time course experiment, cells were trypsinized using isotonic $10 \times$ trypsin for $1 \mathrm{~h}$ and a stack of 30 reference images of both layers of beads was acquired. To obtain traction maps of the whole cell diameter, we merged a series of overlapping images using a correlation-based algorithm. In this case, registration was achieved by equalling displacement fields over the overlapping regions and imposing zero traction outside the colony. All experiments were conducted in the presence of serum, at $37^{\circ} \mathrm{C}$ and $5 \% \mathrm{CO}_{2}$.

Preparation of polyacrylamide gel substrates. Polyacrylamide substrate preparation was adapted from previously published protocols ${ }^{18,37}$ to enable image registration and improve resolution of the displacement fields (see Supplementary Information S2). The concentrations of crosslinker and polymer were adjusted for a Young's modulus of $1.3 \mathrm{kPa}$ (ref. 38). The details of the protocol for polyacrylamide 
gel preparation including all modifications from previous publications are provided below. Step 1: A few drops of $0.1 \mathrm{M} \mathrm{NaOH}$ were added to the centre of each $35 \mathrm{~mm}$ dish (glass bottom, uncoated, no.0; MatTek). The dishes were air-dried overnight. Step 2: 2-3 drops of 97\% 3-aminopropyltrimethoxylsilane were added over the $\mathrm{NaOH}$-stained circular regions from the previous step. The dishes were then washed and the glass surface was scrubbed with a foam swab to remove debris. The dishes were washed again and $400 \mu \mathrm{l}$ of a solution containing $0.0001 \%$ yellow fluorescent carboxylate-modified beads ( $2 \mu \mathrm{m}$ diameter, Fluospheres, Invitrogen) was added to each well. These beads were used for image registration. After the dishes dried, $0.5 \%$ glutaraldehyde in PBS was added to the central region in each dish for $30 \mathrm{~min}$. The dishes were subsequently washed and air-dried overnight. Step 3: $20 \mu \mathrm{l}$ of an acrylamide / bis-acrylamide mixture dissolved in ultrapure water containing $0.01 \%$ of 0.5 - $\mu$ m-diameter red fluorescent carboxylate-modified beads (Fluospheres, Invitrogen), $0.5 \%$ of ammonium persulphate and $0.05 \%$ TEMED (Bio-Rad) was added to the centre of each dish. This gel mixture was covered with glass cover slips (18 mm diameter; VWR). To ensure that all red beads laid in the top plane of the gel, the dishes were centrifuged at 500 r.p.m. for 15 min during gelation. Once polymerization was completed, the cover slips were removed. The surface was activated by adding $225 \mu \mathrm{l}$ of a solution containing $4 \mu \mathrm{M}$ sulphosuccinimidyl-6-(4-azido-2-nitrophenylamino) hexanoate (Sulfo-SANPAH; Pierce) dissolved in 0.1 M HEPES buffer. The dishes were then exposed to ultraviolet light for $10 \mathrm{~min}$, washed twice with $0.1 \mathrm{M}$ HEPES solution, washed once with PBS, coated with $1 \mathrm{ml}$ of type-I collagen solution $\left(0.1 \mathrm{mg} \mathrm{ml}^{-1}\right.$; Inamed Biomaterials) and stored at $4{ }^{\circ} \mathrm{C}$. On the day before seeding the cells, the gels were washed, and incubated overnight with $3 \mathrm{ml}$ of MEM with Earle's salts supplemented with 5\% FBS. Then the gel surface was allowed to dry at room temperature for $2 \mathrm{~h}$ immediately before seeding the cells.

Fourier-transform traction microscopy. A new algorithm of traction microscopy was developed to account for finite substrate thickness and force imbalance within the microscope field of view (see Supplementary Information S3). Gel displacements were computed using correlation-based particle image velocimetry. To reduce systematic biases in subpixel resolution and peak-locking effects, we implemented an iterative process ( $n=4$ iterations) based on a continuous window shift technique ${ }^{39}$. The interrogation windows were $25.6 \mu \mathrm{m}$ on a side and window overlap ranged from $3 / 4$ to $7 / 8$.

Received 18 December 2008; accepted 31 March 2009; published online 3 May 2009

\section{References}

1. Lecaudey, V. \& Gilmour, D. Organizing moving groups during morphogenesis Curr. Opin. Cell Biol. 18, 102-107 (2006).

2. Martin, P. \& Parkhurst, S. M. Parallels between tissue repair and embryo morphogenesis. Development 131, 3021-3034 (2004).

3. Friedl, P. \& Wolf, K. Tumour-cell invasion and migration: Diversity and escape mechanisms. Nature Rev. Cancer 3, 362-374 (2003).

4. du Roure, O. et al. Force mapping in epithelial cell migration. Proc. Natl Acad. Sci. USA 102, 2390-2395 (2005).

5. Vaughan, R. B. \& Trinkaus, J. P. Movements of epithelial cell sheets in vitro J. Cell Sci. 1, 407-413 (1966).

6. Omelchenko, T. et al. Rho-dependent formation of epithelial 'leader' cells during wound healing. Proc. Natl Acad. Sci. USA 100, 10788-10793 (2003).

7. Friedl, P., Hegerfeldt, Y. \& Tusch, M. Collective cell migration in morphogenesis and cancer. Int. J. Dev. Biol. 48, 441-449 (2004).

8. Gov, N. S. Collective cell migration patterns: Follow the leader. Proc. Natl Acad. Sci. USA 104, 15970-15971 (2007).

9. Poujade, M. et al. Collective migration of an epithelial monolayer in response to a model wound. Proc. Natl Acad. Sci. USA 104, 15988-15993 (2007).

10. Liu, C. H. et al. Force fluctuations in bead packs. Science 269, 513-515 (1995).

11. O’Hern, C. S., Langer, S. A., Liu, A. J. \& Nagel, S. R. Force distributions near jamming and glass transitions. Phys. Rev. Lett. 86, 111-114 (2001).

12. Ostojic, S., Somfai, E. \& Nienhuis, B. Scale invariance and universality of force networks in static granular matter. Nature 439, 828-830 (2006).

13. Lauffenburger, D. A. \& Horwitz, A. F. Cell migration: A physically integrated molecular process. Cell 84, 359-369 (1996).

14. Keren, K. et al. Mechanism of shape determination in motile cells. Nature 453, 475-480 (2008).

15. Hu, K. et al. Differential transmission of actin motion within focal adhesions. Science 315, 111-115 (2007).

16. Giannone, G. et al. Lamellipodial actin mechanically links myosin activity with adhesion-site formation. Cell 128, 561-575 (2007).
17. Beningo, K. A. et al. Nascent focal adhesions are responsible for the generation of strong propulsive forces in migrating fibroblasts. J. Cell Biol. 153, 881-888 (2001).

18. Dembo, M. \& Wang, Y. L. Stresses at the cell-to-substrate interface during locomotion of fibroblasts. Biophys. J. 76, 2307-2316 (1999).

19. Montell, D. J. Morphogenetic cell movements: Diversity from modular mechanical properties. Science 322, 1502-1505 (2008).

20. Matsubayashi, Y., Ebisuya, M., Honjoh, S. \& Nishida, E. ERK activation propagates in epithelial cell sheets and regulates their migration during wound healing. Curr. Biol. 14, 731-735 (2004).

21. Bindschadler, M. \& McGrath, J. L. Sheet migration by wounded monolayers as an emergent property of single-cell dynamics. J. Cell Sci. 120, 876-884 (2007).

22. Holmes, S. J. The behaviour of the epidermis of amphibians when cultivated outside the body. J. Exp. Zool. 17, 281-295 (1914).

23. Hutson, M. S. et al. Forces for morphogenesis investigated with laser microsurgery and quantitative modeling. Science 300, 145-149 (2003).

24. Farooqui, R. \& Fenteany, G. Multiple rows of cells behind an epithelial wound edge extend cryptic lamellipodia to collectively drive cell-sheet movement. J. Cell Sci. 118, 51-63 (2005).

25. Butler, J. P., Tolic-Norrelykke, I. M., Fabry, B. \& Fredberg, J. J. Traction fields, moments, and strain energy that cells exert on their surroundings. Am. J. Physiol. Cell Physiol. 282, C595-C605 (2002).

26. Sabass, B., Gardel, M. L., Waterman, C. M. \& Schwarz, U. S. High resolution traction force microscopy based on experimental and computational advances. Biophys. J. 94, 207-220 (2008).

27. Del Alamo, J. C. et al. Spatio-temporal analysis of eukaryotic cell motility by improved force cytometry. Proc. Natl Acad. Sci. USA 104, 13343-13348 (2007).

28. Merkel, R., Kirchgessner, N., Cesa, C. M. \& Hoffmann, B. Cell force microscopy on elastic layers of finite thickness. Biophys. J. 93, 3314-3323 (2007).

29. Fenteany, G., Janmey, P. A. \& Stossel, T. P. Signaling pathways and cell mechanics involved in wound closure by epithelial cell sheets. Curr. Biol. 10, 831-838 (2000)

30. Goffin, J. M. et al. Focal adhesion size controls tension-dependent recruitment of alpha-smooth muscle actin to stress fibers. J. Cell Biol. 172, 259-268 (2006).

31. Saez, A., Buguin, A., Silberzan, P. \& Ladoux, B. Is the mechanical activity of epithelial cells controlled by deformations or forces? Biophys. J. 89 , L52-L54 (2005)

32. Gov, N. S. Modeling the size distribution of focal adhesions. Biophys. J. 91 , 2844-2847 (2006).

33. Zegers, M. M. et al. Pakl and PIX regulate contact inhibition during epithelial wound healing. EMBO J. 22, 4155-4165 (2003).

34. Shraiman, B. I. Mechanical feedback as a possible regulator of tissue growth. Proc. Natl Acad. Sci. USA 102, 3318-3323 (2005).

35. Van Hecke, M. Granular matter: A tale of tails. Nature 435, 1041-1042 (2005).

36. Coppersmith, S. N. et al. Model for force fluctuations in bead packs. Phys. Rev. E 53, 4673-4685 (1996).

37. Wang, N. et al. Cell prestress. I. Stiffness and prestress are closely associated in adherent contractile cells. Am. J. Physiol. Cell Physiol. 282, C606-C616 (2002).

38. Yeung, T. et al. Effects of substrate stiffness on cell morphology, cytoskeletal structure, and adhesion. Cell. Motil. Cytoskeleton 60, 24-34 (2005).

39. Gui, L. \& Wereley, S. T. A correlation-based continuous window-shift technique to reduce the peak-locking effect in digital PIV image evaluation. Exp. Fluids 32, 506-517 (2002).

\section{Acknowledgements}

We thank N. Gavara, R. Sunyer and C. Y. Park for experimental support and D. Tschumperlin and members of the Fredberg laboratory for insightful discussions.

\section{Author contributions}

X.T., J.P.B. and J.J.F. designed research. X.T. and M.R.W. carried out experiments. J.P.B and X.T conducted theoretical analysis. T.E.A., D.A.W. and E.M. contributed to design protocols and data interpretation. X.T., J.P.B. and J.J.F. wrote the manuscript. J.P.B. and J.J.F. oversaw the project.

\section{Additional information}

Supplementary information accompanies this paper on www.nature.com/naturephysics. Reprints and permissions information is available online at http://npg.nature.com/ reprintsandpermissions. Correspondence and requests for materials should be addressed to X.T. or J.J.F. 